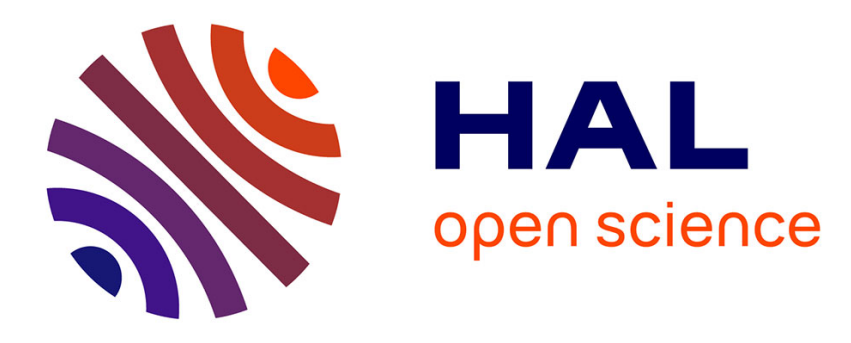

\title{
The interaction of hybrid nanoparticles inserted within surfactant bilayers \\ Doru Constantin
}

\section{To cite this version:}

Doru Constantin. The interaction of hybrid nanoparticles inserted within surfactant bilayers. Journal of Chemical Physics, 2010, 133 (14), pp.144901. 10.1063/1.3499741 . hal-00524842

\section{HAL Id: hal-00524842 \\ https://hal.science/hal-00524842}

Submitted on 8 Oct 2010

HAL is a multi-disciplinary open access archive for the deposit and dissemination of scientific research documents, whether they are published or not. The documents may come from teaching and research institutions in France or abroad, or from public or private research centers.
L'archive ouverte pluridisciplinaire $\mathbf{H A L}$, est destinée au dépôt et à la diffusion de documents scientifiques de niveau recherche, publiés ou non, émanant des établissements d'enseignement et de recherche français ou étrangers, des laboratoires publics ou privés. 


\title{
The interaction of hybrid nano-particles inserted within surfactant bilayers
}

\author{
Doru Constantin ${ }^{1, \text { a) }}$ \\ Laboratoire de Physique des Solides, Université Paris-Sud, CNRS, UMR 8502, 91405 Orsay, \\ France.
}

(Dated: 7 October 2010)

We determine by small-angle X-ray scattering the structure factor of hydrophobic particles inserted within lamellar surfactant phases for various particle concentrations. The data is then analyzed by numerically solving the Ornstein-Zernicke equation, taking into account both the intra- and inter-layer interactions. We find that particles within the same layer repel each other and that the interaction potential (taken as independent of the concentration) has a contact value of $2.2 k_{B} T$ and a range of about $10 \AA$. If the amplitude is allowed to decrease with increasing concentration, the contact value in the dilute limit is about $5 k_{B} T$, for a similar range.

PACS numbers: 82.70.Dd, 87.16.dt, 61.05.cf

Keywords: membrane; inclusions; interaction; X-ray

\section{INTRODUCTION}

The elucidation of membrane-mediated interaction between inclusions in the cell membrane (such as integral proteins or membrane-active antibiotic peptides) is of paramount importance for understanding their biological activity. Since the underlying problem is the organization of the membrane -seen as a two-dimensional complex system- it has become clear over the last three decades that the concepts developed in soft matter physics for the understanding of self-assembled systems are operative in this context and that 'simple' models can yield valuable information.

It is therefore not surprising that sustained theoretical efforts attempted to provide a detailed description of these complex systems; they are either continuumelasticity theories ${ }^{1-4}$ or more detailed models taking into account the molecular structure of the lipid bilayer ${ }^{5-8}$. In the few cases when the models were validated and refined using experimental data, these approaches were often successful. For instance, the lifetime of the gramicidin channel is known to depend on the thickness ${ }^{9}$ and tension ${ }^{10}$ of the membrane; it was shown that a continuum-elasticity model provides a satisfactory description of this phenomenon ${ }^{11}$.

However, this body of theoretical work has not yet been matched by the experimental results, the first of which were obtained by directly measuring the radial distribution function of membrane inclusions using freezefracture electron microscopy $(\text { FFEM })^{12-15}$. These data were compared to liquid state models and could be described by a hard-core model with, in some cases, an additional repulsive or attractive interaction ${ }^{16-18}$. However, FFEM has not been extensively employed since, certainly due to the inherent experimental difficulties; furthermore, the particle distribution observed in the frozen

a)Electronic mail: constantin@lps.u-psud.fr sample is not necessarily identical to that at thermal equilibrium.

A step forward was taken by Huang and collaborators, who showed that the distribution of membrane inclusions within the plane of the layers can be studied using small-angle X-ray and neutron scattering ${ }^{19,20}$. First of all, these techniques are perfectly adapted to the typical length scales to be probed. The measurement is averaged over a large number of particles and over long times, without perturbing the system; thus, one has access to the structure factor of the interacting particles. However, in these studies, at most two peptide-to-lipid concentrations $P / L$ were investigated for each system and no values were given for the interaction potential, the data being explained in terms of purely hard-core interactions.

Building upon this work, we recently studied systems with a varying density of inclusions. This is indispensable since, even though each structure factor (taken separately) can be described by a hard-core model, the apparent radius obtained changes with the concentration, signalling the presence of an additional interaction. For instance, we were able to measure the interaction potential of alamethicin pores in dimyristoylphosphatidylcholine (DMPC) bilayers. We showed that, aside from the expected hard-disk repulsion (with a radius corresponding to the geometrical radius of the pore) the pore interaction exhibits a repulsive contribution, with a range of about $3 \mathrm{~nm}$ and a contact value of $2.4 k_{B} T^{21}$. On the other hand, for gramicidin pores in dilauroyl-phosphatidylcholine (DLPC) bilayers, while the interaction is still repulsive, the parameter values are quite different, with a higher contact value and much shorter range. In the latter case, the interaction was shown to decrease with the pore concentration, in agreement with the hydrophobic matching model ${ }^{22}$. These results are in qualitative agreement with recent theoretical predictions ${ }^{7,23}$ and can be used as a test for other theoretical and numerical results.

The biological molecules cited above act specifically by insertion within cell membranes, so their affinity for lipid bilayers is assured, which is a significant advantage. 
However, their use as membrane probes has important shortcomings: their X-ray scattering contrast is low and the positions of the constitutive atoms are not always well-defined (membrane proteins can adopt various conformations as a function of the environment, while the pores formed by antimicrobial peptides often comprise variable numbers of monomers). These features contribute to the difficulty of obtaining high-quality scatering data on membranes with inclusions.

Fortunately, if one is interested in the general physical properties of membranes seen as two-dimensional complex fluids, rather than in the behaviour of a specific active molecule, other inclusions can be used. For instance, we have shown recently that tin oxo-clusters can be inserted within surfactant bilayers and that their interaction potential can be determined ${ }^{24}$.

These types of hybrid organic-inorganic particles have the following advantages:

- They are perfectly monodisperse (actually isomolecular) and "rigid" (their atomic configuration is well-defined).

- Their scattering contrast is high (due to the presence of metal atoms).

- Their surface properties can be tailored by changing the nature of the peripheral groups.

The purpose of this article is to develop a full analysis of the system, beyond the initial approach used in Ref. 24. Two main improvements can be noted:

- We were able to determine the complete structure factor, $S\left(q_{r}, q_{z}\right)$, while the initial study was only concerned with the equatorial slice, $S\left(q_{r}, q_{z}=0\right)$. This required new experimental data, for $q_{z} \neq 0$.

- We employed a more elaborate statistical model (based on the numerical solution of the OrnsteinZernicke equation with the Percus-Yevick closure) to relate the structure factor to the interaction potential; our first approach was based on the random-phase approximation (RPA).

We have also checked the reproducibility of the results (see Appendix A), by comparing the old and new data, thus verifying both the reliability of the method and the stability of the samples.

\section{MATERIALS AND METHODS}

We used as inclusions hybrid nanoparticles consisting of a tin oxide core decorated with butyl chains. The complete formula is: $\left\{(\mathrm{BuSn})_{12} \mathrm{O}_{14}(\mathrm{OH})_{6}\right\}^{2+}(4-$ $\left.\mathrm{CH}_{3} \mathrm{C}_{6} \mathrm{H}_{4} \mathrm{SO}_{3}^{-}\right)_{2}$, shortened to BuSn12 in this paper. The synthesis and structural details are given in Ref. 25. The BuSn12 particles were dissolved in ethanol prior to use.
The membranes were composed of dimethyldodecylamine-N-oxide (DDAO), a singlechain zwitterionic surfactant. The DDAO (purchased from Sigma-Aldrich) was first dried in vacuum for $20 \mathrm{~h}$ to remove any residual water ${ }^{26}$ and then dissolved in isopropanol.

The two stock solutions were then mixed to yield the desired BuSn12/DDAO ratio and the mixtures dried in vacuum, yielding a final mass of about $200 \mathrm{mg}$ for each sample. Varying amounts of water were then added so that the mixtures were in the fluid lamellar $L_{\alpha}$ phase $^{26}$. For DDAO, the molecular weight is 229.40 , the density is $0.84 \mathrm{~g} / \mathrm{cm}^{3}$ and the bilayer thickness is $25 \pm 1 \AA^{27,28}$, resulting in an area per surfactant molecule $A_{\mathrm{DDAO}}=$ $37.8 \AA^{2}$. The molecular weight of the BuSn12 is 2866.7, with a density of $1.93 \mathrm{~g} / \mathrm{cm}^{3} 25$. The (two-dimensional) number density of particles in the plane of the membrane, $n$, is calculated using the data above and neglecting the possible increase in bilayer surface due to the inclusions.

The lamellar phases were then drawn into flat glass capillaries (VitroCom Inc., Mt. Lks, NJ), $100 \mu \mathrm{m}$ thick and $2 \mathrm{~mm}$ wide by aspiration with a syringe and the capillaries were flame-sealed. Good homeotropic alignment (lamellae parallel to the flat faces of the capillary) was obtained by thermal treatment, using a Mettler FP52 heating stage. The samples were heated up to the isotropic phase (at $130^{\circ} \mathrm{C}$ ) and then cooled down to the lamellar phase at a rate of $1^{\circ} \mathrm{C} / \mathrm{min}$.

We studied the samples by small-angle X-ray scattering (SAXS), on the the bending magnet beamline BM02 $(\mathrm{D} 2 \mathrm{AM})^{29}$ of the European Synchrotron Radiation Facility (ESRF, Grenoble, France). The photon energy was $11 \mathrm{keV}$ and the sample-to- detector distance about $27 \mathrm{~cm}$, with a scattering vector in the range $0.04<q<0.9 \AA^{-1}$. The detector is a charge-coupled device Peltier-cooled camera (SCX90-1300, from Princeton Instruments Inc., NJ) with a detector size of $1340 \times 1300$ pixels. Data preprocessing (dark current subtraction, flat field correction, radial regrouping and normalization) was performed using the bm2img software developed at the beamline. The scattering geometry is discussed in section IV.

\section{MODEL AND ANALYSIS}

Since the particles are identical in shape, the scattering intensity can be written as the product of a form factor (only depending on the internal constitution of the particles) and a structure factor, which describes the interaction between particles ${ }^{30}: I(\boldsymbol{q})=S(\boldsymbol{q}) \cdot|F(\boldsymbol{q})|^{2}$, with:

$$
S\left(q_{z}, q_{r}\right)=\frac{1}{N}\left\langle\left.|| \sum_{k=1}^{N-1} \exp \left(-i \boldsymbol{q} \boldsymbol{r}_{k}\right)\right|^{2}\right\rangle
$$

where $N$ is the number of objects and object " 0 " is taken as the origin of the coordinates. 
We expect the form factor of the particles to be dominated by their inorganic core, as the electron density of the butyl chains is similar to that of the dodecyl chains within the bilayers and to that of ethanol. This assumption is confirmed by the intensity at higher scattering vectors $\left(q>0.5 \AA^{-1}\right)$, which is well described for all samples by the form factor of a sphere $|F f(R, q)|^{2}$, with a radius (used as a free fitting parameter) $R=4.5 \pm 0.2 \AA$, in good agreement with the average radius of the tin oxide "cage" estimated from the crystallographic data.

Dividing the measured intensity by the form factor above yields the structure factor $S(\boldsymbol{q})$. Standard error propagation then yields the uncertainty, but the resulting values (corresponding to the statistical error) are much smaller than the discrepancy between the fitting model and the experimental data (the goodness-of-fit function $\chi^{2}$ per data point is much larger than one). Recognizing that this discrepancy is mostly due to systematic errors in data acquisition, background subtraction etc. and also possibly to the inadequacy of the theoretical models, we assign to each data point a fixed uncertainty $\sigma$; in the following, we take for definiteness $\sigma=0.05$, which is a rough estimate of the difference between model and data, insofar it yields $\chi^{2}$ values of unit order.

Determining the structure factor for systems composed of many particles interacting via a known potential is one of the fundamental endeavors of liquid state theory.

Two cases must be considered:

- The situation when the particles are dissolved in a simple solvent will act as a "reference state"; more precisely, it will allow us to check whether the "naked" particles can be described simply by a hard core repulsion or whether an additional term must be considered and what its characteristics are.

- The most interesting situation is of course that of particles inserted within the surfactant bilayers, which will be the main result of our study. Due to the anisotropic nature of the matrix, the analysis must account for the difference between the interaction between particles contained within the same layer and that from one layer to the next.

\section{A. Structure factor of an isotropic solution}

In solution, the interaction between particles is taken as isotropic, described by a two-body potential depending only on the distance between their centers: $V_{3 D}(\boldsymbol{r})=$ $V_{3 D}(r)$. Consequently, the structure factor $S(q)$ is equally isotropic, depending only on the magnitude of the wave vector $q$.

Using as input the interaction potential, the structure factor was determined numerically by solving iteratively the Ornstein-Zernicke equation with the Percus-Yevick closure condition. The algorithm is based on the method of Lado ${ }^{31,32}$; see Appendix B for the details. The in-
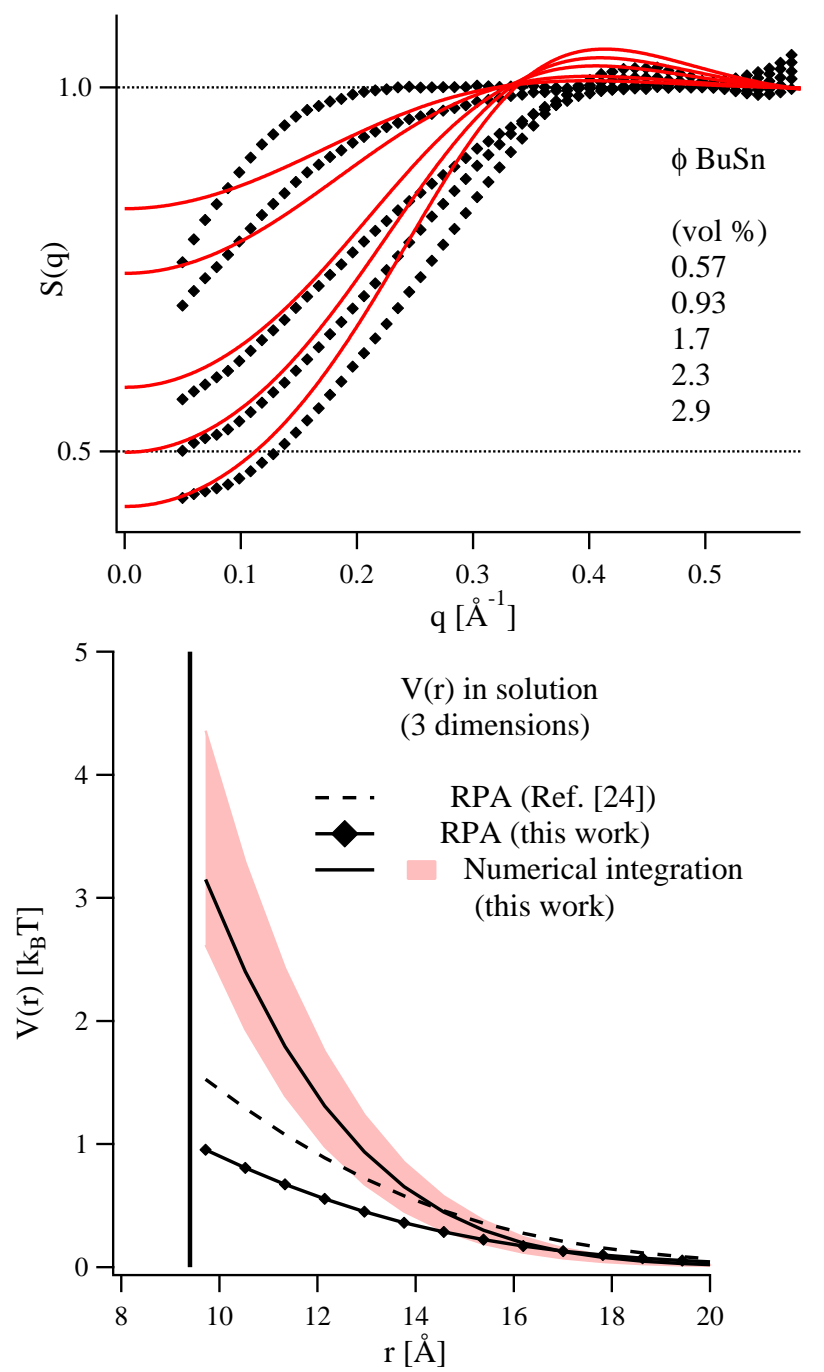

FIG. 1. Top: Experimental three-dimensional structure factors for BuSn12 particles in ethanol (symbols) and fits with the Gaussian model discussed in the text (solid lines) for five volume concentrations $\phi . S(q)$ decreases with increasing $\phi$. Bottom: Interaction potential obtained from the data above via the numerical procedure discussed in Section III A (solid line and shaded area for the uncertainty range) and within the RPA approximation (solid line and symbols). For comparison, we also show the RPA results obtained in Ref. 24 (dashed line).

teraction potential is described by a hard core with diameter $2 R=9.4 \AA$ and an additional "soft" component, modelled either as a Gaussian centered at the origin ${ }^{24}$ : $V(r)=U_{0} \exp \left[-\frac{1}{2}\left(\frac{r}{\xi}\right)^{2}\right] \quad r \geq 2 R$ or as a decreasing exponential with contact value $U_{c}$ and decay range $\xi$ : $V(r)=U_{c} \exp [-(r-2 R) / \xi] \quad r \geq 2 R$. 


\section{B. Structure factor in the lamellar phase}

The phase (and hence the structure factor) is now anisotropic. We assume that there is no in-plane ordering (the particles form a $2 \mathrm{D}$ liquid in the plane of the bilayer), so that $S$ only depends on the absolute value of the in-plane scattering vector $q_{r}=\sqrt{q_{x}^{2}+q_{y}^{2}}$ and on the scattering vector along the membrane normal $q_{z}$.

For an ideal gas (no interaction) $S=1$, while if the particles interact only in the plane of the bilayer and not from one bilayer to the next, $S=S\left(q_{r}\right)$ only. In the general case, the structure factor can be developed $\mathrm{as}^{20}$ :

$$
S\left(q_{r}, q_{z}\right)=S_{0}\left(q_{r}\right)+2 \sum_{m=1}^{\infty} \cos \left(q_{z} d m\right) S_{m}\left(q_{r}\right)
$$

with each partial structure factor $S_{m}$ describing the interaction between particles situated $m$ layers away:

$$
S_{m}\left(q_{r}\right)=\delta_{0 m}+2 \pi \rho \int_{0}^{\infty} r \mathrm{~d} r J_{0}\left(r q_{r}\right)\left[g_{m}(r)-1\right]
$$

where $m \geq 0$ and $g_{m}(r)$ is the (normalized) probability of finding a particle at a distance $r$ in bilayer $m$, knowing that a particle is present at the origin in bilayer 0 .

This expansion merely reflects the discrete nature of the stack along the $z$ direction. It is especially convenient since generally the interaction does not extend very far along $z$. Note also that Equation (2) is only valid for the geometrically perfect case when the distance between neighboring layers is rigorously $d$. In practice, this distance varies due to thermal fluctuations and to frozen-in defects, leading to a smearing of the diffraction pattern at high $q_{z}$ values. We describe this effect phenomenologically by a Lorentzian factor. Finally, we use:

$$
S\left(q_{r}, q_{z}\right)=S_{0}\left(q_{r}\right)+2 \frac{\cos \left(q_{z} d\right)}{1+\left(q_{z} \sigma\right)^{2}} S_{1}\left(q_{r}\right)
$$

where the disorder parameter $\sigma$ has units of length. For thermal fluctuations, $\sigma^{2}=\left\langle\left(z_{1}-z_{0}\right)^{2}\right\rangle \simeq \eta(d / \pi)^{2}$, where $\eta$ is the Caillé parameter.

As we will see below, the structure factor $S_{1}\left(q_{r}\right)$ due to nearest-bilayer interaction has a localized peak. For convenience, we describe it by a Gaussian function:

$$
S_{1}=A_{1} \exp \left[-\frac{\left(q-q_{\max }\right)^{2}}{2 \Delta q^{2}}\right]
$$

The partial structure factors $S_{0}(q)$ and $S_{1}(q)$ are similar to those describing a two-dimensional binary mixture $\mathrm{AB}$, with the formal identification $S_{0}=S_{A A}=S_{B B}$ and $S_{1}=S_{A B}$. As we will see below, these two components are enough to describe the experimental data, so there is no need to go beyond $m=1$ (interaction between adjacent bilayers).
The (experimentally determined) functions $S_{0}(q)$ and $S_{1}(q)$ can be described -using well-established results in liquid state theory- in terms of the interaction potentials $V_{0}(r)$ (between particles within the same layer) and $V_{1}(r)$ (between adjacent layers). The derivation of the model and the implementation details are given in Appendix B.

\section{RESULTS}

\section{A. Interaction in solution}

The 3D structure factors of BuSn12 particles in ethanol are shown in Figure 1 (top) for five different concentrations (lines and symbols). They are fitted with the Gaussian interaction model discussed in Section III A, yielding $U_{0}=15 k_{B} T$ and $\xi=5.1 \AA$, such that the potential at contact is $U_{c}=3.5 k_{B} T$. The fits are shown as solid lines. The corresponding interaction potential is shown in Figure 1 (bottom) as solid line. The shaded area represents the uncertainty (see Appendix B 3 for details). For comparison, we show in the same figure the potential obtained in the RPA approximation for the current data (solid line and symbols) and for that in Ref. 24 (dashed line). The exponential model yields very similar results, in terms of goodness of fit and in potential shape for parameter values $U_{c}=4.5 k_{B} T$ and $\xi=2.1 \AA$.

\section{B. Interaction between layers}

In order to quantify the interaction between the layers (along the smectic director $\hat{z}$ ), one needs access to the complete structure factor $S\left(q_{r}, q_{z}\right)$. This is achieved by using the experimental configuration described in Figure 2, as first discussed in Ref. 20: the incoming X-ray beam (with wave vector $\boldsymbol{k}_{i}$ ) is incident upon the flat capillary at an angle $\alpha$ to its normal $\boldsymbol{n}$, which coincides with the smectic director $\hat{z}$. A point on the $2 \mathrm{D}$ detector uniquely defines an outgoing wave vector $\boldsymbol{k}_{o}$ for the scattered signal, and thus a scattering vector given by: $\boldsymbol{q}=\boldsymbol{k}_{o}-\boldsymbol{k}_{i}$. One can therefore assign to each pixel values for $q_{z}=\boldsymbol{q} \cdot \hat{z}$ and $q_{r}=\left|\boldsymbol{q}-q_{z} \hat{z}\right|$.

The raw scattering image is then re-gridded to an appropriate region of the reciprocal space, $\left(q_{r}, q_{z}\right)$. For each pixel in the target space, the algorithm identifies the corresponding point in the starting image, and the closest 9 pixels are averaged to yield the final intensity value. It is easily shown that the accessible range in reciprocal space is triangle-shaped, with an angle $2 \alpha$ at the origin. Finally, the resulting image is divided by the form factor of the particle to yield the structure factor.

As an illustration, we show in Figure 2 re-gridded data at four different incidence angles $\alpha$ for the sample with $n=1.97610^{-3} \AA^{-2}$. A modulation along $q_{z}$ is clearly visible, indicating the presence of the $S_{1}$ component and hence of an interaction between layers. The images also display a linear slope in $q_{z}$, probably due to imperfect 

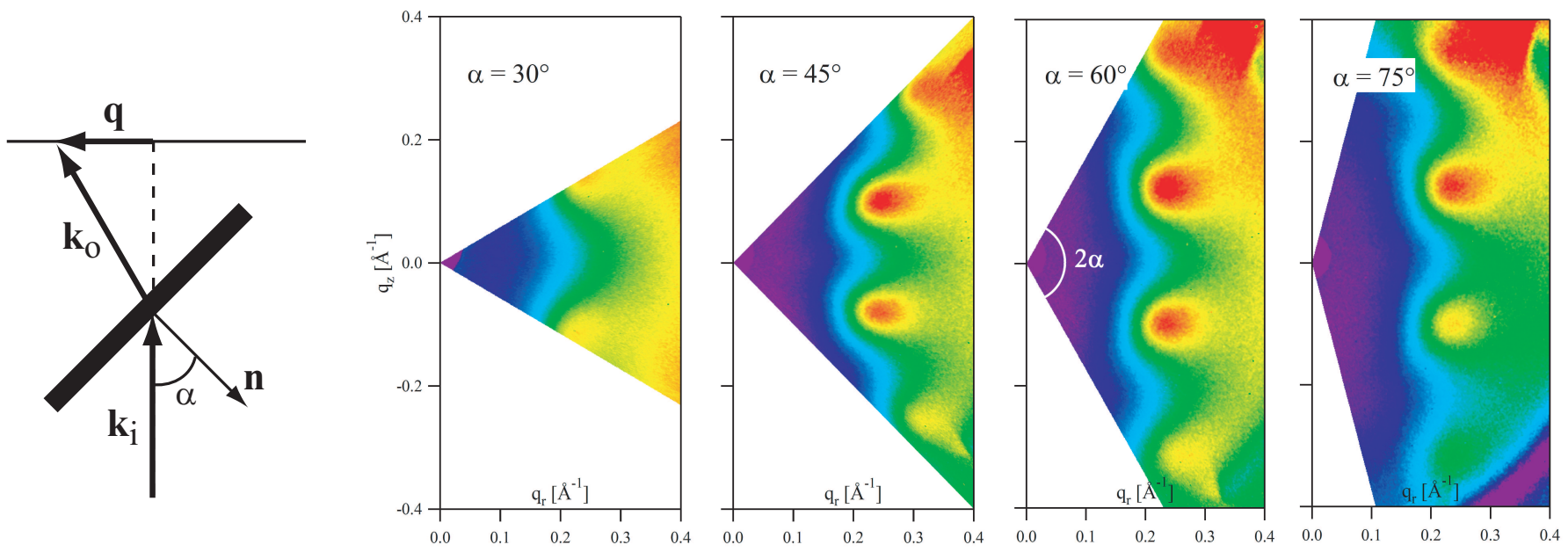

FIG. 2. Left: Sketch of the experimental configuration. Right: Re-gridded scattering data for various values of the incident angle $\alpha$ (indicated within the images).

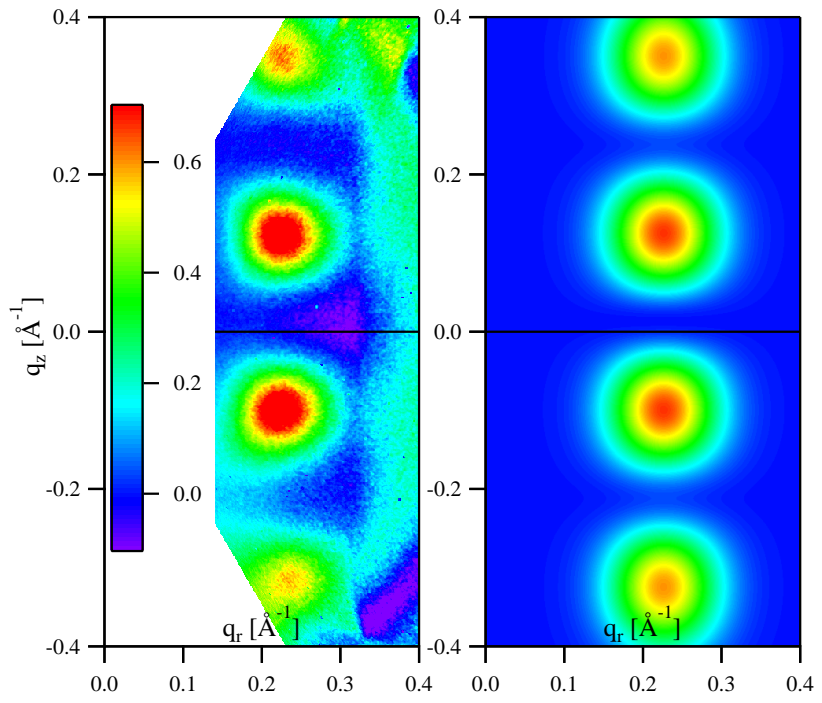

FIG. 3. Comparison between the experimental data for $S\left(q_{r}, q_{z}\right)-S\left(q_{r}, q_{z}=0\right)$ derived from the image taken at $\alpha=60^{\circ}$ in Figure 2 and the model (4).

background subtraction. This artefact is removed prior to further treatment.

Although the physically relevant functions are $S_{0}\left(q_{r}\right)$ and $S_{1}\left(q_{r}\right)$, the most easily accessible quantity is the "equatorial" cut through reciprocal space $S_{\text {eq }}=$ $S\left(q_{r}, q_{z}=0\right)=S_{0}+2 S_{1}$ obtained under normal incidence $\left(\alpha=0\right.$ in Figure 2). We therefore subtract $S_{\text {eq }}$ from the re-gridded data (an example is shown in Figure 3, left) and fit it with an appropriately modified version of Equation (4). The best fit for the data in Figure 3 is given in the right image.

The vertical streak on the right in the experimental data is due to an imperfectly subtracted diffuse scatter-

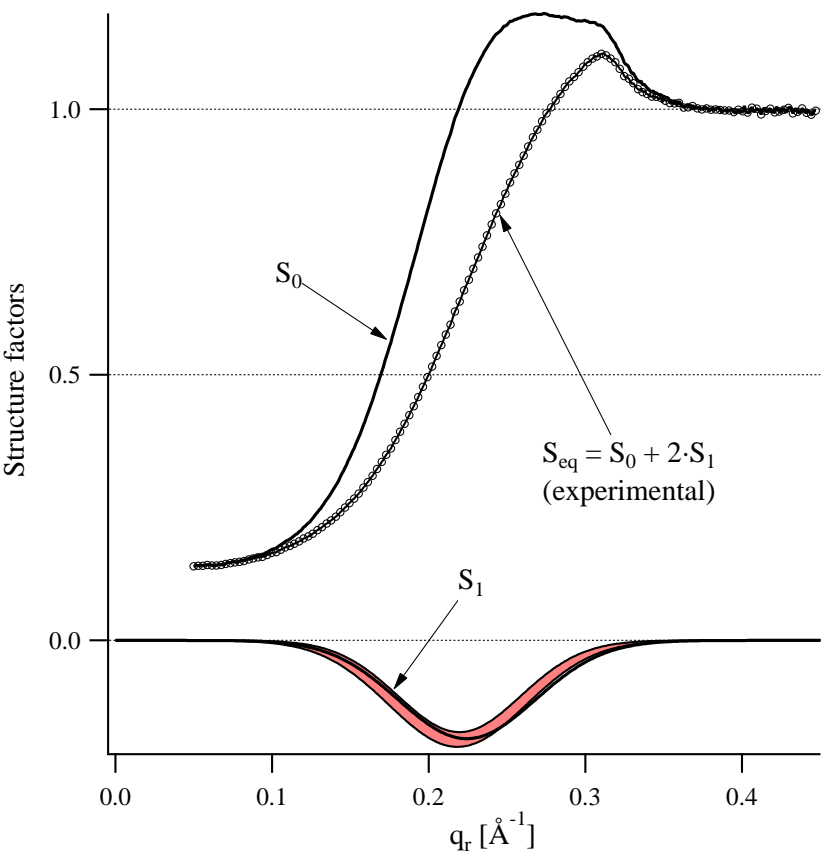

FIG. 4. Partial structure factors for the sample with $n=$ $1.97610^{-3} \AA^{-2}$, extracted from the data in Figure 2. Symbols: the equatorial structure factor $S_{\text {eq }}$ (experimental data). Lines: the structure factors $S_{0}$ and $S_{1}$. For $S_{1}$, the solid line represents the estimate extracted from the configuration with $\alpha=60^{\circ}$, while the shaded area around the curve represents the uncertainty (see text).

ing ring due to a kapton window in the beam (we found that proper background subtraction is quite difficult for tilted samples, i.e. for $\alpha \neq 0$ ). Otherwise, the agreement between data and the fit is good, showing that the model is accurate (in particular, there is no need to include higher-order partial structure factors). 
This treatment yields $S_{1}\left(q_{r}\right)$, which is then subtracted from the equatorial structure factor $S_{\text {eq }}\left(q_{r}\right)$ to yield $S_{0}\left(q_{r}\right)$. Figure 4 shows these functions for the most concentrated sample (extracted from the data in Figure 2). In the case of $S_{1}$, the solid line represents the best fit to scattering data taken for $\alpha=60^{\circ}$ (Figure 3), while the shaded area contains the curves obtained for $\alpha=45,60$ and $75^{\circ}$, providing a rough estimate of the experimental uncertainty.

\section{Interaction within the layer}

Once the experimental data for the partial structure factors $S_{0}\left(q_{r}\right)$ and $S_{1}\left(q_{z}\right)$ (or, equivalently, $S_{\text {eq }}$ and $S_{1}$ ) is available, it can be described in terms of the interaction potentials $V_{0}(r)$ and $V_{1}(r)$ via well-known methods in the theory of liquids, presented in Appendix B. The main goal of this work is determining the membrane-mediated potential $V_{0}(r)$. The interbilayer interaction $V_{1}(r)$, although needed for a complete analysis, is inessential and, furthermore, is probably very sensitive to the swelling of the phase. We therefore use a single functional form for it, given by a linear decrease from a maximum $U_{1}$ when the particles have the same in-plane position $\boldsymbol{r}$ to 0 when they are a distance $\xi_{1}$ away. After extensive tests we found that, for various shapes of the in-plane potential $V_{0}(r)$, the best fit for $V_{1}(r)$ is described by an amplitude $U_{1}$ around $1 k_{B} T$ and a range $\xi_{1}$ of about $25 \AA$.

In the following, we concentrate on the parameters describing $V_{0}(r)$. We considered several functional forms, such as an exponential or a linear decrease, a Gaussian centered at the origin or at the contact and a complementary error function. The best result for each of them is shown in Figure 5, except for the linear decrease which yields much worse fits.

We obtained very good results for the Gaussian centered at contact: $V_{0}(r)=U_{c} \exp \left[-\frac{1}{2}\left(\frac{r-2 R}{\xi}\right)^{2}\right]$ (solid line in Figure 5; the corresponding structure factors are plotted in Figure 6, along with the experimental data) with fit parameters $U_{c}=2.2 \pm 0.2 \mathrm{k}_{\mathrm{B}} \mathrm{T}$ and $\xi=$ $10.8 \pm 0.8 \AA$ while the hard disk radius $R$ is fixed at $4.7 \AA$. The shaded area corresponds to the fit uncertainty, estimated as in Appendix B 3.

\section{Hydrophobic matching}

In the treatment above, we considered that the interaction potential is independent of the particle concentration. However, one can infer from a simple elastic theory (and confirm by experimental investigations ${ }^{22}$ ) that the amplitude of the interaction decreases with the concentration of inclusions; briefly, inserting a new particle is easier when the bilayer is already deformed by existing inclusions. Accounting for this effect requires introduc-

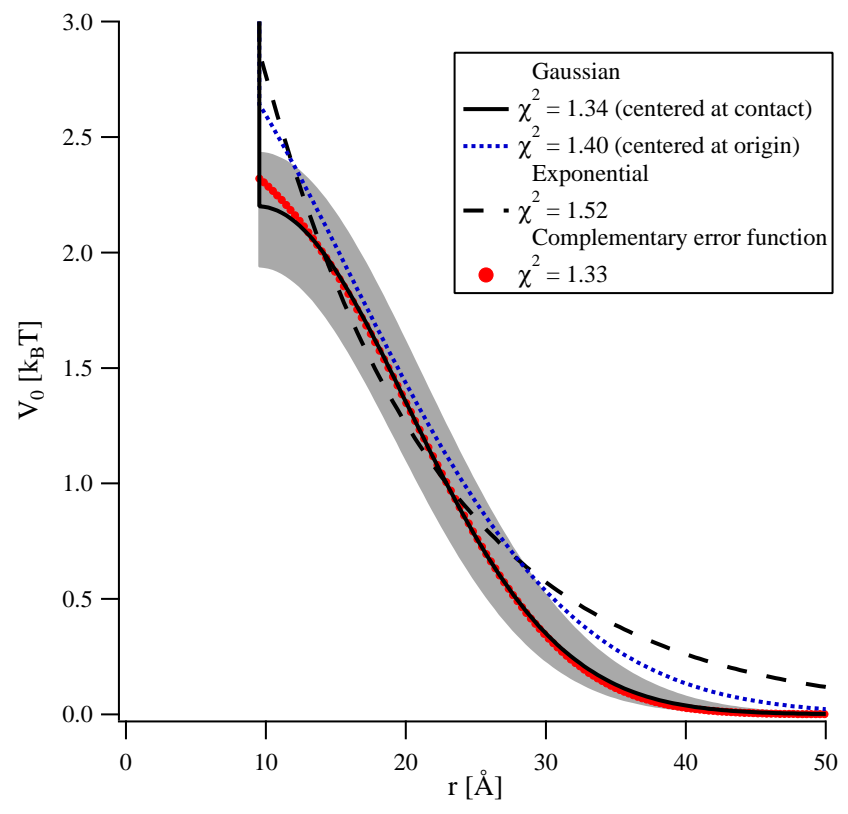

FIG. 5. Best results for some of the functional forms used for $V_{0}(r)$ and corresponding $\chi^{2}$ values. For the Gaussian centered at contact, we also plot the uncertainty range obtained as described in Appendix B 3.

ing a new fit parameter, the concentration $n_{0}$, for which the interaction decreases significantly. We will consider the simplest model, whereby the membrane deformation is linear (and thus the elastic energy is quadratic) in the concentration: $V_{0}(n, r)=V_{0}(0, r)\left(1-n / n_{0}\right)^{2}$ for $n<n_{0}$ and $V_{0}(n, r)=0$ for $n \geq n_{0}$. We take $V_{1}(r)$ as independent of the concentration. As before, the in-plane potential is described by a Gaussian centered at contact. The fit is significantly improved, yielding $\chi^{2}=1.00$, for parameters $U_{c}=5.0 \pm 0.6 \mathrm{k}_{\mathrm{B}} \mathrm{T}, \xi=9.3 \pm 0.7 \AA$ and $n_{0} \simeq 510^{-3} \AA^{-2}$ (roughly twice the highest experimental value available).

We emphasize that the values presented above correspond to the highly diluted limit $V_{0}(0, r)$, i.e. to the interaction between two particles in the absence of any other; this is the relevant case for comparison with most theoretical and numerical models.

One can see this decrease of the interaction potential between two particles due to the concentration (i.e. to the presence of other particles) as a crude way of accounting for many-body effects. However, this approximation is not controlled and its accuracy can only be verified by more complete theoretical models or by numerical simulations ${ }^{33}$.

\section{v. DISCUSSION AND CONCLUSION}

The main results of this work are the in-plane interaction potential of the BuSn12 particles, $V_{0}(r)$ in the lamellar phase and $V(r)$ in solution; they are summarized in 


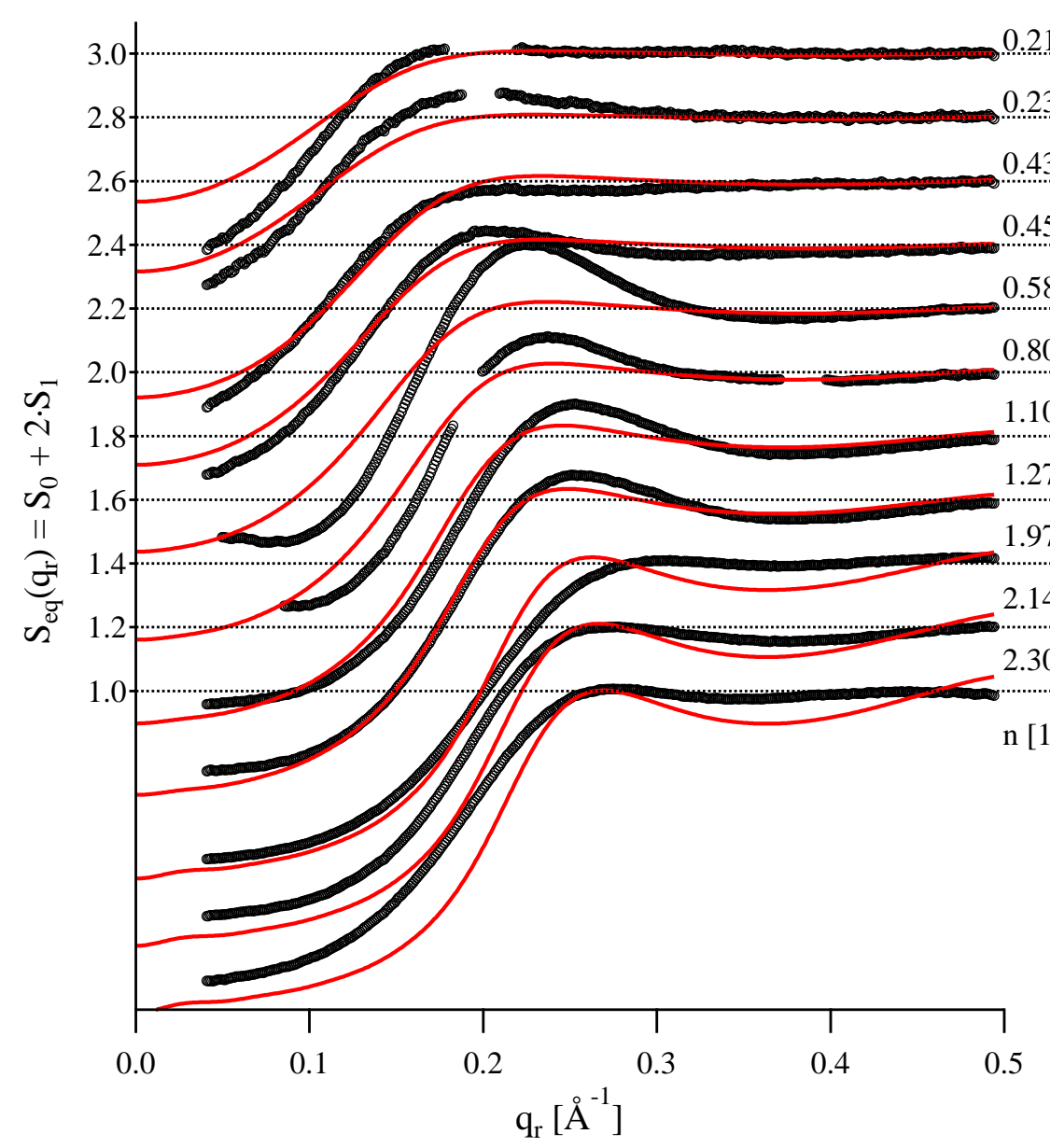

0.217

0.231

0.434

0.451

0.584

0.801

1.102

1.275

1.976

2.147

2.304

$n\left[10^{-3} \AA^{-2}\right]$

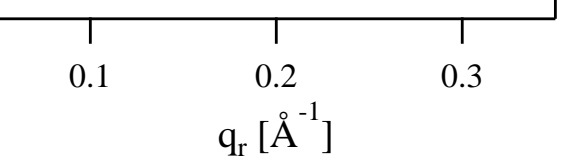

FIG. 6. Best fits (lines) to the experimental data (symbols) with the model described in Section IV C. $V_{0}(r)$ is a Gaussian centered at contact (solid line in Figure 5.)

Figure 7. One can see straightaway that the uncertainty is small compared to the amplitude of the curves and to the difference between them, except very close to contact.

The qualitative conclusions to be drawn are similar to those already obtained in Reference 24 using a simplified model, although the amplitudes are somewhat different. The interaction potential in solution is shortranged (about $5 \AA$ from contact), but at short distances it becomes stronger than the in-plane repulsion estimated with the constant-amplitude model $\left(4 k_{B} T\right.$, as opposed to $2.2 k_{B} T$ at contact). Notwithstanding the uncertainty and the non-additivity of the potentials, it is tempting to speculate on a possible non-monotonic dependence of the "effective" membrane-mediated interaction, defined by subtracting the interaction in solution from that in the lamellar phase.

\section{Appendix A: Reproducibility}

Since the signals to be measured are fairly weak and unstructured, the question of their reproducibility is of the utmost importance. We therefore measured a few samples twice (about one year apart), in the same experimental conditions (see Section II), and in normal incidence, so that only the equatorial structure factor $S_{\text {eq }}\left(q_{r}\right)$ is available. The results are shown in Figure 8 (left). Gray lines: earlier data, used in the analysis of Ref. 24. Black lines: more recent data, measured on the same samples. For clarity, the curves are shifted vertically in steps of 0.25 .

Notwithstanding the discrepancy close to the peak position, the agreement is quite good, especially at small angles where $S$ departs significantly from 1 . Indeed, this is the range where the interaction potential plays a significant role. For coherence, the comparison is done using the same simplified treatment as in Ref. 24, i. e. neglecting the interaction between layers $V_{1}(r)$ and obtaining the Fourier transform $V\left(q_{r}\right)$ of the remaining interaction potential $V_{0}(r) \equiv V(r)$ via the RPA approximation; see Ref. 24 for the details.

The two sets of data for $V\left(q_{r}\right)$ are shown in Figure 8 (right). The solid line and the shaded area correspond to the results of Ref. 24 (average value and uncertainty) 


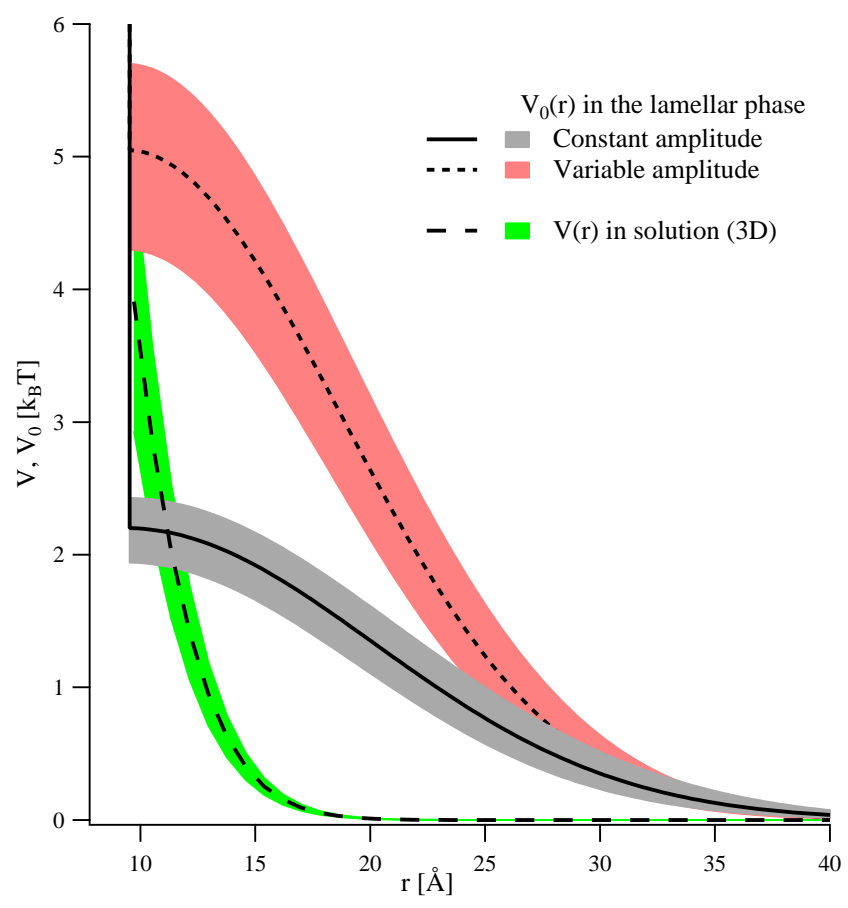

FIG. 7. Best results for the in-plane interaction potential $V_{0}(r)$ in the lamellar phase, with constant amplitude (solid line - see Section IV C) and with variable amplitude (dotted line - see Section IV D) as well as for the interaction potential $V(r)$ in solution (dashed line - see Section IV A.) The shaded areas represent the uncertainty, determined as explained in Appendix B 3.

while the symbols are obtained directly from the more recent values of $S_{\mathrm{eq}}\left(q_{r}\right)$ (black lines in the left panel). Although the new data seems to yield a stronger interaction than the old, the difference is within the estimated uncertainty range. We conclude that the measurement method is reliable and that the samples did not age significantly over a period of one year.

\section{Appendix B: Interaction model}

\section{Analytical development}

The interaction is treated in the framework of the Ornstein-Zernike relation, with the Percus-Yevick closure approximation. The structure factors $S_{m}(q)$ are computed using the method introduced by Lado ${ }^{31,32}$. An implementation for the case of no interbilayer interaction has already been used ${ }^{22}$. In the present case, we extend the model to the case of several interacting bilayers. As discussed in the main text, the system can be mapped to a multi-component fluid, where each component corresponds to particles inserted within one bilayer and all particles formally occupy the same twodimensional space. Throughout this analysis, only the two-body interaction is taken into account.
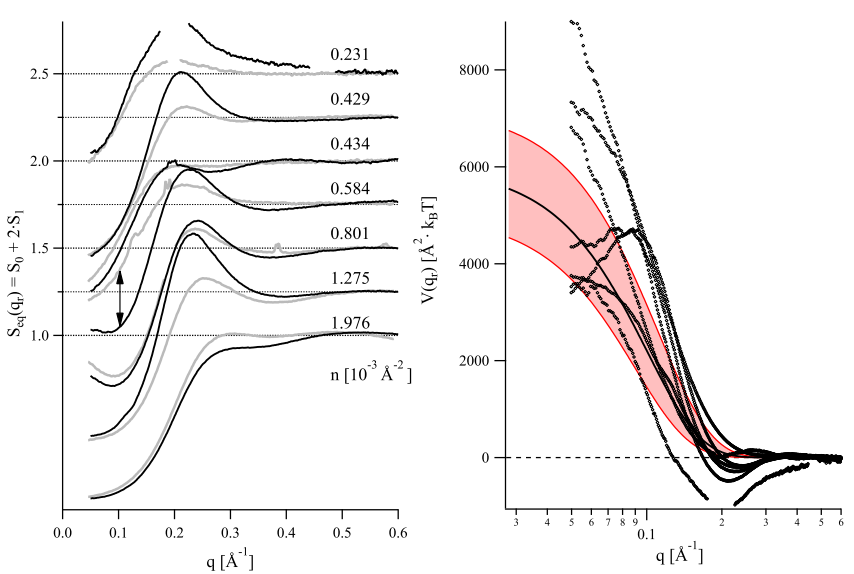

FIG. 8. Left: comparison between the equatorial structure factor $S_{\mathrm{eq}}\left(q_{r}\right)$ determined in two different experimental runs (black and gray curves). A noticeable difference appears for $n=0.584$, signaled by the double-headed arrow. Right: comparison between the corresponding potentials $V\left(q_{r}\right)$, estimated via the RPA approximation.

This system has been studied for a long time ${ }^{34,35}$. In the following, we will use the notations of Ref. 35, except for the interaction potential, denoted here by $V$ (instead of $\phi$ ) for coherence with previous work. We consider $n$ bilayers (indexed by lowercase Greek indices) with periodic boundary conditions (bilayer $n$ is identical to bilayer $0)$. The relevant equations are:

The definition of the total correlation function $h(r)$ in terms of the radial distribution function $g(r)$ :

$$
h_{\alpha \beta}(r)=g_{\alpha \beta}(r)-1
$$

The Ornstein Zernike relation, defining the direct correlation function $c(r)$ :

$$
h_{\alpha \beta}(r)=c_{\alpha \beta}(r)+\sum_{\gamma} \rho_{\gamma} \int \mathrm{d}^{2} s c_{\alpha \gamma}(|\boldsymbol{s}|) h_{\gamma \beta}(|\boldsymbol{r}-\boldsymbol{s}|)
$$

where $\rho_{\gamma}=\rho$ is the number density in the plane of the bilayers and $\gamma$ runs over all bilayers. Alternatively, the equation above can be rewritten in reciprocal space, yielding:

$$
h_{\alpha \beta}(q)=c_{\alpha \beta}(q)+\sum_{\gamma} \rho_{\gamma} c_{\alpha \gamma}(q) h_{\gamma \beta}(q)
$$

The Percus-Yevick closure:

$$
c_{\alpha \beta}(r)=\left[1+h_{\alpha \beta}(r)\right]\left[1-\exp \left(\frac{V_{\alpha \beta}(r)}{k_{B} T}\right)\right]
$$

with $V_{\alpha \beta}(r)$ representing the interaction potential between particles situated in bilayers $\alpha$ and $\beta$. For simplicity's sake, we consider that the particles only interact within the same bilayer and with particles in the nearestneighbor bilayers $\left(V_{\alpha \beta}(r) \equiv 0\right.$ for $\left.|\alpha-\beta|>1\right)$. Furthermore, translation symmetry (an identical environment 
for all bilayers, i.e. periodic boundary conditions) and mirror symmetry $\left(V_{\alpha \beta} \equiv V_{\beta \alpha}\right.$, and similar relations for functions $c$ and $h$ ) allow us to define all functions with respect to layer 0 , i.e. $\alpha=0$ throughout.

The equations above must be solved numerically; for convergence reasons, it is much better to work with the indirect correlation function (denoted in Ref. 31 by $H(r)), \gamma_{0 \beta}(r)=h_{0 \beta}(r)-c_{0 \beta}(r)$ instead of the total correlation function.

As an example, for $n=3$ bilayers (with periodic boundary conditions, $3 \equiv 0$ ), equations (B3) and (B4) can be written out explicitly as:

$$
\begin{aligned}
& \gamma_{00}(q)=\rho\left[c_{00}\left(\gamma_{00}+c_{00}\right)+2 c_{01}\left(\gamma_{01}+c_{01}\right)\right] \\
& \gamma_{01}(q)=\rho\left[c_{00}\left(\gamma_{01}+c_{01}\right)+c_{01}\left(\gamma_{00}+c_{00}\right)+c_{01}\left(\gamma_{01}+c_{01}\right)\right]
\end{aligned}
$$

$c_{00}(r)=\left[1+\gamma_{00}\right]\left[\exp \left(-V_{00} / k_{B} T\right)-1\right]$

$c_{01}(r)=\left[1+\gamma_{01}\right]\left[\exp \left(-V_{01} / k_{B} T\right)-1\right]$

where we omitted the argument of the functions on the right-hand side. One starts by applying in real space equations (B5c-d) with reasonable initial guesses, and then solving (B5a-b) in reciprocal space. The procedure is iterated until stability is reached. The experimentally relevant structure factors are simply: $S_{0}(q)=1+h_{00}(q)$ and $S_{1}(q)=h_{01}(q)$. For completeness, the solution of $(\mathrm{B} 5 \mathrm{a}-\mathrm{b})$ is:

$$
\begin{aligned}
& \gamma_{00}(q)=\frac{-c_{00}^{2}\left(c_{00}+c_{01}-1 / \rho\right)+2 c_{01}^{2}\left(c_{00}+1 / \rho\right)}{\left(c_{00}-1 / \rho\right)^{2}+c_{01}\left(c_{00}-1 / \rho\right)-2 c_{01}^{2}} \\
& \gamma_{01}(q)=c_{01} \frac{-\left(c_{00}+c_{01}\right)\left(c_{00}-1 / \rho\right)+c_{00} / \rho+2 c_{01}^{2}}{\left(c_{00}-1 / \rho\right)^{2}+c_{01}\left(c_{00}-1 / \rho\right)-2 c_{01}^{2}}
\end{aligned}
$$

We checked that, for relevant values of the interaction parameters, the structure factors obtained with $n=5$ and $n=7$ are almost superposed, and slightly different from those obtained for $n=3$ (with periodic boundary conditions in all cases). All results presented above are obtained with $n=7$. We also checked that the higher order structure factors are negligible, viz. $S_{3}(q) \ll S_{2}(q) \ll$ $S_{1}(q)$, in agreement with the experimental observations.

\section{Software implementation}

The routine discussed above is implemented in IGOR PRO 6 as an "all-at-once" function, i.e. all points of the output vector are returned at the same time. This approach is particularly useful for such iterative procedures, where, in order to get its value at one point, the entire function needs to be calculated anyway. The Fourier transform was implemented as a matrix operation with pre-computed coefficients over a fixed equidistant grid.
Wherever possible, the wave operations were done using the MatrixOp command.

For each combination of fit parameters, the structure factors $S_{0}$ and $S_{1}$ are calculated for all densities. Comparison with the experimental data yields the goodnessof-fit function $\chi^{2}$, which is minimized using the Optimize operation with the simulated annealing method.

\section{Confidence range}

Once a minimum is found, the goodness-of-fit function $\chi^{2}$ is plotted as a function of the parameters $U_{0}$ (or $U_{c}$ ) and $\xi$.

For the 3D case (interaction in solution), one notices that the minimum is in fact an extended valley, covering a wide range of parameters, and roughly defined as the locus of the points where the integral of the potential is constant. This is understandable, since the fit is mainly sensitive to the low values of the scattering wave vector $q$, where it is affected by $V(q \rightarrow 0)$, i.e. by the integral of $V(r)$, rather than by its finer details. We therefore choose a number of points along this valley, with close to minimum $\chi^{2}$, and trace the corresponding $V(r)$ instances. The confidence range (shown as a shaded area in Figure 1, bottom) is chosen by manually adjusting the parameters to yield lower and higher envelopes to this sheaf of curves.

In the 2D case (interaction within the layer), the minimum is well localized in the $\left(U_{c}, \xi\right)$ plane. However, a standard statistical estimation of the confidence range is irrelevant, since it would yield extremely tight confidence ranges. Indeed, the discrepancy between model and data is mainly due to systematic errors and (presumably) to an inexact functional form for the interaction potential. In order to account for the latter effect we took as an acceptable increase $\Delta \chi^{2}$ the difference between the Gaussian centered at contact $\left(\chi^{2}=1.34\right)$ and that centered at the origin $\left(\chi^{2}=1.40\right)$, both plotted in Figure 5 . The uncertainty on $U_{c}$ and $\xi$ given in Section IV C is determined based on this numerical value. For comparison, we also show the exponential decay with $\chi^{2}=1.52$. We follow a similar procedure for the variable amplitude model discussed in Section IV D, with $\Delta \chi^{2}=0.1$.

\section{ACKNOWLEDGMENTS}

The ESRF is gratefully acknowledged for the award of beam time (experiment 02-01-756) and we thank C. Rochas for competent and enthusiastic support. F. Ribot is acknowledged for providing the BuSn12 particles and for edifying discussions. 
${ }^{1}$ N. Dan, P. Pincus, and S. Safran, "Membrane-induced interactions between inclusions," Langmuir, 9, 2768 (1993).

${ }^{2}$ N. Dan, A. Berman, P. Pincus, and S. Safran, "Membraneinduced interactions between inclusions," J. Phys. II France, 4, 1713 (1994).

${ }^{3}$ H. Aranda-Espinoza, A. Berman, N. Dan, P. Pincus, and S. Safran, "Interaction between inclusions embedded in membranes," Biophys. J., 71, 648 (1996).

${ }^{4}$ K. Bohinc, V. Kralj-Iglič, and S. May, "Interaction between two cylindrical inclusions in a symmetric lipid bilayer," J. Chem. Phys., 119, 7435 (2003)

${ }^{5}$ S. Marčelja, "Lipid-mediated protein interaction in membranes," Biochim. Biophys. Acta, 455, 1 (1976).

${ }^{6} \mathrm{~T}$. Sintes and A. Baumgärtner, "Protein attraction in membranes induced by lipid fluctuations," Biophys. J., 73, 2251 (1997).

${ }^{7}$ P. Lagüe, M. J. Zuckermann, and B. Roux, "Lipid-mediated interactions between intrinsic membrane proteins: A theoretical study based on integral equations," Biophys. J., 79, 2867 (2000).

${ }^{8}$ S. May and A. Ben-Shaul, "A molecular model for lipid-mediated interaction between proteins in membranes," Phys. Chem. Chem. Phys., 2, 4494 (2000).

${ }^{9}$ J. R. Elliott, D. Needham, J. P. Dilger, and D. A. Haydon, "The effects of bilayer thickness and tension on gramicidin singlechannel lifetime," Biochim. Biophys. Acta, 735, 95 (1983).

${ }^{10}$ M. Goulian, O. N. Mesquita, D. K. Fygenson, C. Nielsen, O. S. Andersen, and A. Libchaber, "Gramicidin channel kinetics under tension," Biophys. J., 74, 328 (1998).

${ }^{11} \mathrm{H}$. W. Huang, "Deformation free energy of bilayer membrane and its effect on gramicidin channel lifetime," Biophys. J., 50, 1061 (1986).

${ }^{12}$ B. A. Lewis and D. M. Engelman, "Bacteriorhodopsin remains dispersed in fluid phospholipid bilayers over a wide range of bilayer thicknesses," J. Mol. Biol., 166, 203 (1983).

${ }^{13}$ Y. S. Chen and W. L. Hubbell, "Temperature- and lightdependent structural changes in rhodopsin-lipid membranes," Exp. Eye Res, 17, 517 (1973).

${ }^{14} \mathrm{R}$. James and D. Branton, "Lipid- and temperature-dependent structural changes in Acholeplasma laidlawii cell membranes," Biochim. Biophys. Acta, 323, 378 (1973).

${ }^{15}$ J. R. Abney, J. Braun, and J. C. Owicki, "Lateral interactions among membrane proteins: Implications for the organization of gap junctions," Biophys. J., 52, 441 (1987).

${ }^{16}$ L. T. Pearson, B. A. Lewis, D. M. Engelman, and S. I. Chan, "Pair distribution functions of bacteriorhodopsin and rhodopsin in model bilayers," Biophys. J., 43, 167 (1983).

${ }^{17}$ L. T. Pearson, J. Edelman, and S. I. Chan, "Statistical mechanics of lipid membranes, protein correlation functions and lipid ordering," Biophys. J., 45, 863 (1984).

${ }^{18}$ J. Braun, J. R. Abney, and J. C. Owicki, "Lateral interactions among membrane proteins: Valid estimates based on freezefracture electron microscopy," Biophys. J., 52, 427 (1987).

${ }^{19}$ T. A. Harroun, W. T. Heller, T. M. Weiss, L. Yang, and H. W. Huang, "Experimental evidence for hydrophobic matching and membrane-mediated interactions in lipid bilayers containing gramicidin," Biophys J., 76, 937 (1999).

${ }^{20}$ L. Yang, T. Weiss, T. Harroun, W. Heller, and H. Huang, "Supramolecular structures of peptide assemblies in membranes by neutron off-plane scattering: Method of analysis," Biophys. J., 77, 2648 (1999).

${ }^{21}$ D. Constantin, G. Brotons, A. Jarre, C. Li, and T. Salditt, "Interaction of alamethicin pores in DMPC bilayers," Biophys. J., 92, 3978 (2007)

${ }^{22}$ D. Constantin, "Membrane-mediated repulsion between gramicidin pores," Biochim. Biophys. Acta, 1788, 1782 (2009).

${ }^{23}$ P. Lagüe, M. J. Zuckermann, and B. Roux, "Lipid-mediated interactions between intrinsic membrane proteins: Dependence on protein size and lipid composition," Biophys. J., 81, 276 (2001).

${ }^{24}$ D. Constantin, B. Pansu, M. Impéror, P. Davidson, and F. Ribot, "Repulsion between inorganic particles inserted within surfactant bilayers," Phys. Rev. Lett., 101, 098101 (2008).

${ }^{25}$ C. Eychenne-Baron, F. Ribot, N. Steunou, C. Sanchez, F. Fayon, M. Biesemans, J. C. Martins, and R. Willem, "Reaction of butyltin hydroxide oxide with $p$-toluenesulfonic acid: Synthesis, $\mathrm{x}$-ray crystal analysis, and multinuclear NMR characterization of $\left\{(\mathrm{BuSn})_{12} \mathrm{O}_{14}(\mathrm{OH})_{6}\right\}\left(4-\mathrm{CH}_{3} \mathrm{C}_{6} \mathrm{H}_{4} \mathrm{SO}_{3}\right)_{2}$," Organometallics, 19, 1940 (2000).

${ }^{26} \mathrm{~V}$. Kocherbitov and O. Söderman, "Hydration of dimethyldodecylamine-n-oxide: Enthalpy and entropy driven processes," J. Phys. Chem. B, 110, 13649 (2006).

${ }^{27}$ G. Orädd, G. Lindblom, G. Arvidson, and K. Gunnarsson, "Phase equilibria and molecular packing in the N,Ndimethyidodecylamine oxide/gramicidin $\mathrm{D} /$ water system studied by $2 \mathrm{H}$ nuclear magnetic resonance spectroscopy," Biophys. J., 68, 547 (1995).

${ }^{28}$ P. Wästerby and P.-O. Quist, "Interactions between membranes crammed with gramicidin," Langmuir, 14, 3704 (1998).

${ }^{29}$ J. P. Simon, S. Arnaud, F. Bley, J. F. Bérar, B. Caillot, V. Comparat, E. Geissler, A. de Geyer, P. Jeantey, F. Livet, and H. Okuda, "A new small-angle x-ray scattering instrument on the french CRG beamline at the ESRF multiwavelength anomalous scattering/diffraction beamline (D2AM)," J. Appl. Cryst., 30, 900 (1997).

${ }^{30}$ P. M. Chaikin and T. C. Lubensky, Principles of Condensed Matter Physics (Cambridge University Press, 1995).

${ }^{31} \mathrm{~F}$. Lado, "Pressure-consistent integral equation for classical fluids: Hard-sphere solutions," J. Chem. Phys., 47, 4828 (1967).

${ }^{32} \mathrm{~F}$. Lado, "Equation of state of the hard-disk fluid from approximate integral equations," J. Chem. Phys., 49, 3092 (1968).

${ }^{33}$ T. A. Harroun, W. T. Heller, T. M. Weiss, L. Yang, and H. W. Huang, "Theoretical analysis of hydrophobic matching and membrane-mediated interactions in lipid bilayers containing gramicidin," Biophys. J., 76, 3176 (1999).

${ }^{34}$ J. L. Lebowitz, "Exact solution of generalized Percus-Yevick equation for a mixture of hard spheres," Phys. Rev., 133, A895 (1964).

${ }^{35}$ R. J. Baxter, "Ornstein-Zernike relation and Percus-Yevick approximation for fluid mixtures," J. Chem. Phys., 52, 4559 (1970). 